\title{
Shifted Comprehension and Psycho-semiotic Distinctions of Its Means
}

\author{
Leonid Tchertov \\ Art School, St. Petersburg, Russia
}

\begin{abstract}
The paper contains a theoretical analysis of communicative means comprehended at diverse levels of psyche. The comprehension is understood as an involving of received information into internal systems of cognition, valuation, and skills of the subject. Together with the objects directly given for the feeling, the objects represented by various semiotic means are comprehended as well. Such indirect, or shifted, comprehension can occur at different levels and be realized not only with help of verbalized logical concepts, but also by "infralogical" images and schemes. Particularly, along with ideograms and pictograms as the means intended for upper levels of interpretation, one can speak also about perceptograms and even sensograms aimed for comprehension at perceptual and sensorial levels, correspondingly. The norms of regular connections between the means of comprehension from diverse its levels can be considered as codes with representative and communicative functions. Depending on the psychical levels, to which elements and structures of their expression and content planes belong, one can distinguish a number of psycho-semiotic types of these codes.
\end{abstract}

Keywords: shifted comprehension at diverse levels, codes, perception, recognition, understanding

\section{Introduction: Shifted Comprehension as a Subject of Psycho-semiotic Studies}

The subject of the presented paper is the shifted comprehension of non-verbal semiotic means - an ability of the mind to interpret some presented objects as the means of representation of other objects that are not given directly for sensations. The comprehension is understood in a wide sense as the inclusion of any data received from the outside into the systems of knowledge, values, or skills, which the subject already possesses. Correspondingly, the comprehension has various modes and can be not only cognitive activity aimed to knowledge of objects in images and logical concepts, but also projective, connected with creation of behavior plans or valuative, related with affective reactions to images and plans. Each of these modes contains also various levels of psychical activity and can be represented by communicative means. The subject of this paper is limited by connections of these means with cognitive levels of the comprehension.

In this aspect, it is possible to distinguish direct comprehension aimed at the knowledge of a presented object itself and comprehension shifted to some other object represented with its help. Any representation of objects realized by the communicative means is based on such shifted comprehension. In the same time, its semiotic means can be distinguished depending on the cognitive levels actualized for the shifted comprehension.

The discussed subject belongs together to psychology of cognitive and communicative processes and to semiotics as well. Their collaboration helps to illuminate a field that remains not enough clear for each of them

Leonid Tchertov, Lecturer, Ph.D., Department of Culture, Art School. 
separately. Although a research of communicative and representative means belongs to the sphere of semiotics, the last uses as a rule the theoretical models developed by logicians and linguists that are limited by studying of conventional (arbitrary, non-motivated) signs and not always are sufficient for description of such means of communication as, for example, drawings or sculptures. In these cases, a subject of communication is not verbalized concepts (or not only they), but also images as results of perception and recognition.

For a correct description of this process, it is essential to distinguish the upper levels of semiosis realized by conventional signs developed in human culture and its lower signal-indexical levels that are available even to animals, although can be also transformed by culture (Tchertov, 2017, II. 2). Semiotics in this sphere should enter in union with psychology-despite of the famous thesis about the complete independence of semiotic systems on the "psychical substance" of expression and of content (Hjelmslev, 1953, § 13). In its turn, the psychology that has the images of diverse levels in its traditional field should involve the semiotic concepts for more exact description of the means of their communication. Therefore, the united psycho-semiotic approach gives more hope for a successful study of this subject.

\section{Psychological Levels of Comprehension}

A contribution of psychology into this research is connected foremost with the distinction of cognitive levels, on which the incoming information is processed by a subject. Indeed, the inclusion of the external data in the internal processes of knowledge can occur at its different levels and involve not only verbal-logical understanding, but also non-verbal recognition and perception at "infralogical" levels of the psychical activity (in terms of: Piaget \& Inhelder, 1959). In particular, the visual system works, according to Russian psychologist Boris Ananiev, on three levels: sensorial, perceptual, and apperceptual ones (Ananiev, 1977, p. 127). For example, a compact complex of color shades that has fallen into the field of visual sensations on sensorial level can be unfolded at the perceptual level into the visual image of a three-dimensional colored object located in the external space. If this perceived object is related to a visual scheme of recognition retrieved from memory or if even such scheme is actualized only in imagination, the apperceptual level of cognition is involved as well. This visual scheme can be used without of naming the perceived object by a word, but if such naming occurs, and the more, if a logical category by that is applied, the verbal-logical categorization of different degrees of generalization and levels is added to work of the visual system.

If the comprehension is understood as an inclusion of received data in a system of subject's internal activity, then already a prehension of certain color distinctions in the visual field or tactile sensations in a part of body are initial forms of the comprehension. Really, they are not just fixation of some external data, but also are their correlation with the subject's internal body scheme (left, right, top, bottom, etc.), inclusion into a definite part of the visual field (to the center, to the periphery, etc.). A fortiori, a creation by the subject on basis of these sensorial data of perceptual images of external objects occurs at such level of comprehension that involves these objects in a picture of the perceptible world already irrespective of their relations to the body scheme of the subject (cp. distinction of visual field and visible world in: Gibson, 1950). The polysemantic word perception will be treated below in its narrow sense as a process transforming a complex of incoming sensorial data into a more or less detailed image of external objects that remains relatively constant at different positions to the human body.

However, the perceived (in such narrow sense) object can remain unrecognized or can be recognized in a different way. This recognition is performed at the next level of comprehension. At this level, diverse perceived 
objects are brought under a common scheme and joint with some other known objects as bearers of definite functions, for example, as food, tools, toys, books, etc. As far as this scheme is used without a verbalized concept, it remains in the "infralogical" field of psychical activity. Obviously, that participation of verbal language and logical concepts make the process of comprehension more complete. Nevertheless, it is quite possible to immediately orient oneself in a new environment, without creating its verbal description.

It is true that the upper levels influence the selection and interpretation of the relevant elements and structures of the lower levels. However, this fact does not change the distinction of the mental levels of comprehension, and each of them can in different degree participate in the entire process. If a figure from the Rorschach test is interpreted as, say, "a butterfly", an influence of actualized scheme of its recognition on the created image, is probably more than by the vision of a real butterfly or its naturalistic picture. Each of them creates internal models the world detailed in different degrees and diverse situations need the models detailed variously. While a fast recognition of functioning objects does not demand looking at its details, thorough drawing of the same object, on the contrary, needs to distinguish proportions, lightening, foreshortening, and other peculiar properties that depend on point of view, and therefore it demands to distinguish perceptual images of one the same object.

\section{Types of Shifted Comprehension}

The levels of comprehension that are distinguished in the direct modus are in a similar way differed in cases of shifted comprehension. Each of these levels can be a ground, where not only a direct impression from a presented semiotic means is formed, but also a shifted comprehension of some objects represented by these means. Thereby, the shifting of the mental image as its referral to the object hidden from the direct perception is also realized by the psychic processes of various levels.

Each sign supposes both direct and indirect comprehension. As yet Augustine has defined, each sign is a thing, which influences on feeling and is able to refer thinks to something else (Augustine, 1995, I.2.2, II.1.1). The direct perception and recognition of sign are the conditions for its indirect interpretation that relates the think of a subject to a represented object. This interpretation is an actualization of the sign meaning -its shifted understanding differed from the direct comprehension of a communicative means as a sign.

However, the shifted comprehension is not limited by understanding of conventional signs only and can occur at other levels of mental activity as well. Looking at a marble bust, one can recognize in it, for example, a head of Julius Caesar. This is a mental act of another type, than an understanding of a word Caesar, because in this case instead of conventional connection between a name and its denotat, a visual scheme of recognition a persona is actualized and becomes a means of its representation. If in case of a name interpretation, one can speak about its shifted understanding; in case of the marble bust interpretation, one can speak about shifted recognition. This displaced recognition differs from the direct recognition of a thing in front of a viewer as a piece of marble. Both of these ways of recognition-direct and shifted ones - quite can co-exist by the comprehension of a visible form. In the same time, the perception (in the narrow sense) of the sculpture remains direct, because it reproduces the spatial forms of the same three-dimensional piece of marble.

Another case has place in process of perceiving two-dimensional depictions. In this case, a direct perception of a flat picture as a complex of color paints putted on a surface differs from a shifted perception of a depicted three-dimensional space that is opened for a view "behind" of this surface. The depiction can 
represent something that differs from itself, as the conventional signs also do, and it in a similar way shifts a subject's image to a represented object. However, unlike the conventional signs, a drawing or a photo not only points to these objects, but also shows them, evoking their indirect perception. Such shifted perception differs also from the shifted recognition of a sculpture with help of another recognition scheme than a direct scheme of a marble block. The narrow understood perception of some depicted forms and their spatial location, as well as a perception of real spatial objects, does not need their recognition. Although usually recognition accompanies creation of perceptual images, the cases of perception of some non-recognized objects, persons, or landscapes are also quite possible. Such perception is only a creation of image of a certain spatial configuration, but not obligatory demands of its relation to a scheme of a familiar object. Some masters of drawing recommend even as if to forget the meanings of portrayed objects to draw more accurately their spatial forms (Faworski, 1988, p. 297). Obviously, the shifted perception is unequal also to the shifted understanding of conventional signs as means of nomination or signification of represented objects. In the same time, both a shifted perceptual image of another space and the direct perceptual image of the picture itself as a surface painted by colors can co-exist and are essential for the esthetic valuation of an art piece.

It is possible to speak even on shifted sensations that can co-exist with direct ones. They are possible, for example, when combinations of color paints stimulate appearance of a sensorial image of another color. This effect is a usual method of pointillist paintings (Georges Seurat, Paul Signac, etc.), where an optical mix, for example, of yellow and blue paints finely deposited on canvas gives a sensation of green, mix of blue and red is viewed as violet, etc. The intermodal connections in diverse kinds of synesthesia ("color" sounds, "sounding" colors, etc.) are also the cases of the shifted sensations.

Thus, together with conventional signs interpreted at the conceptual level of the mind, there are communicative means that suppose the interpretation not in a system of logical concepts, but by infralogical ideas, perceptual and sensorial images. Accordingly, communicative means differ depending on the level of psychic interpretation to which they are oriented.

Particularly, in the field of visual communication, the ideograms can be defined as pure conventional visual signs interpreted in the norm at the upper conceptual level of the mind - as, for example, ideograms: 0 or $\infty$. Unlike them, the pictograms are the visual signs that in the norm are related to interpretation at the lower, infralogical level, where a scheme of recognition of a represented object has to be actualized (as, for example, the pictogram: \&). One can speak also on perceptograms stimulating a formation of perceptual images of represented objects - as in cases of diverse pictures. It is possible also to distinguish the sensograms as visual indexes stimulating certain synesthetic quasi-sensory images.

These levels of visual communication can be extended to other cases. One can generally distinguish the ideographic, pictographic, perceptographic, and sensographic levels of interpretation depending on a level of psychological processes that should be actualized by one or another communicative means. Thus, there are shifted understanding, shifted recognition, shifted perception, and shifted sensation related to cases of ideograms, pictograms, perceptograms, and sensograms, correspondingly.

\section{Relations to Peirce's Types of Signs}

The difference of comprehension levels and corresponding semiotic means partially coincides with the distinction between the types of representation in the most famous triad proposed in the semiotics of Charles S. Pierce - the distinction of symbolic, index, and iconic signs (Peirce, 1932). In particular, those semiotic means 
that Pierce defines as symbols always need the direct recognition and a shifted understanding, because to grasp their meaning, there is not enough perception, recognition, or direct categorization of a given sign as a "sign" or as a "word". In the same time, the comprehension of indices and iconic means of representation can be made at other levels of psyche. Some indexes always participate in direct or shifted recognition and many icons can stimulate the shifted perception. However, the connection between the called types of comprehension and Peirce's types of representation is not so unique, because each of these groups has different grounds. If the Peirce's triad is based by definition on the various relations of a representamen to the represented objects, the types of shifted comprehension of semiotic means are the ways of their interpretation by a subject; each of them is an "interpretant", in Percean terms. Therefore, the members of both these groups can be combined in diverse ways.

Particularly, the category of icon connected by Peirce with such way of representation, which is based on similarity between a representamen and represented object, can be correlated not only with the shifted perception of flat depictions, but also with the shifted recognition of three-dimensional sculptures, maquettes, dummies, and other artificial objects that are nevertheless perceived directly. In cases of diagrams, an iconicity takes a part in virtue already not external, but internal structural similarity with a represented object. In this case, no more neither shifted perception nor shifted recognition, but only a shifted understanding is actualized-like in the interpretation of conventional signs. Even mathematical formulas contain, by Peirce, some iconicity in relation to denoted objects, and thereby, their interpretation combines this iconicity with the shifted understanding of conventional signs contained in the formula. Thus, the representation of objects by iconic means uses diverse mechanisms of mental activity and involves diverse cognitive levels of shifted comprehension.

In a similar way, indexes that, by definition, have some direct connections with the represented object also can be combined with diverse types of the shifted comprehension. They evoke the quasi-sensory feelings in cases of synesthesia and other ways of shifted sensations. Together with iconic means, indexes participate also in stimulation of the shifted perception of depicted objects. Such means of depiction as shortening of equal sizes in depth, convergence in a point at a pictorial surface of those lines that represent parallels in the depicted space, etc., are based not on iconicity of depicting forms with depicted objects, but on regular deviations from it. These deviations functioning as indexes of certain spatial relations between depicted objects are systematically introduced in the depiction for just to intensify a pictorial illusion. The indexes participate also in cases of shifted recognition, because the actualization of a suitable scheme of represented object depends on presentation of enough number of features as indexes of appropriate type of objects. At least, a signification of a represented object by a conventional sign is in many cases an actualization of a logical concept that covers a class of objects with certain number of properties as indexes pointing to members of this class.

Even the semiotic means that are defined in the Peirce's classifications of signs as symbols save their connections with different ways of shifted comprehension. Although their interpretation as conventional signs is performed always at the level of shifted understanding, they can in the same time be interpreted at other level of comprehension as semiotic means of other types. For instance, the conventional sign of infinity $\infty$ can be interpreted not only at logical, but also at infralogical levels - as a pictogram of the mythical Ouroboros (a snake biting its own tail) or even can be developed in a detailed perceptogram intended to comprehension already at level of shifted perception. 


\section{Combinations of the Shifting Ways}

The last example shows a possibility to combine diverse ways of the shifted comprehension by interpretation of one the same meaningful object. There are also diverse combinations of the shifting types. An allegorical sculpture is a combination of a shifted recognition with a shifted understanding at conceptual level; a pictogram can be considered not only as a means of evoking a shifted recognition, but also as its combination with shifted perception and with shifted understanding, etc.

In the called cases, one the same object is interpreted by different ways that are added to each other and works together. There are also the cases, when the diverse parts of an object are comprehended by different ways. For example, a flattening of volumes in the reliefs formed at least from the time of Renaissance by the means of the linear perspective is aimed for the shifted perception, as it is in cases of planar depictions formed on the rules of the perspective. In the same time, the relief remains a domain of sculpture intended to shifted recognition that is related especially to most convex parts. A combination of both ways of shifted comprehension takes a place, particularly, in the reliefs made by Lorenzo Ghiberti for the eastern gate of the baptistery of San Giovanni in Florence (see Figure 1). Here the shortenings of the bottom and of heavily flattened buildings in the background stimulate a shifted perception, like drawing; in the same time, the direct perception of much more convexly sculpted figures of the foreground is complemented by shifted recognition of their shapes. (It is clear that a photo at the plane can evoke only shifted perception of the relief).

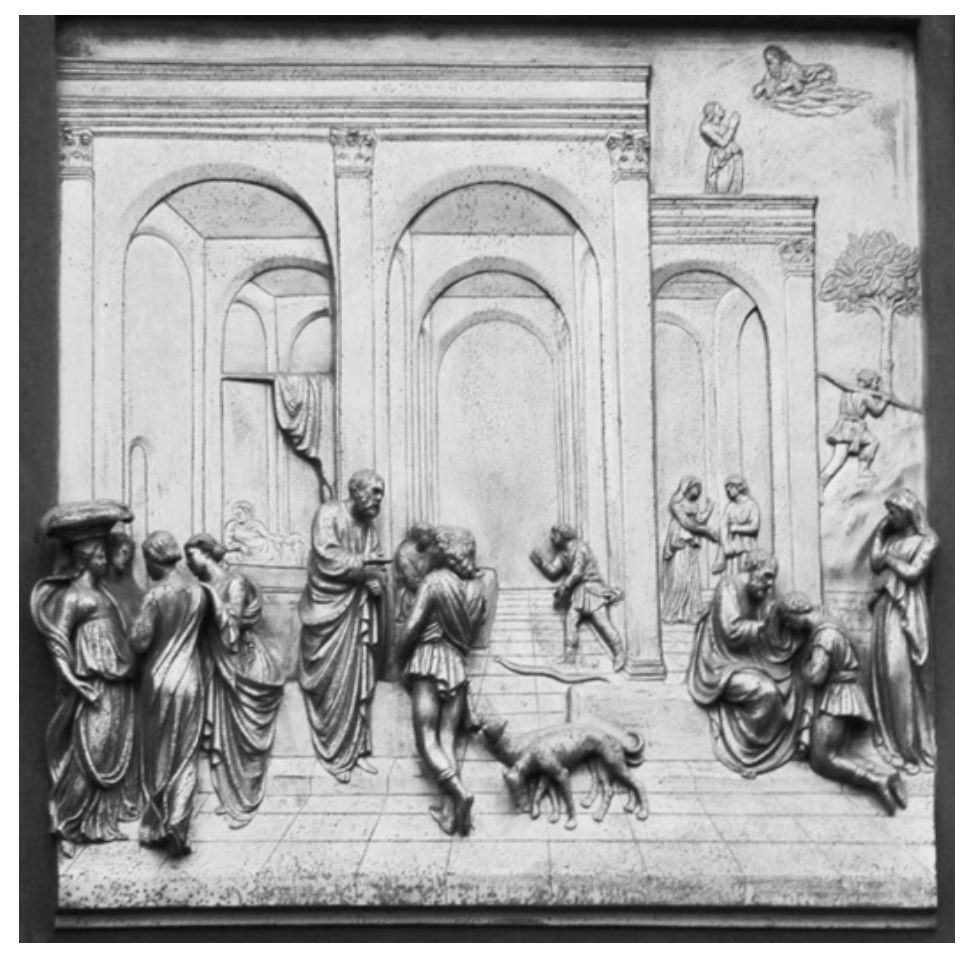

Figure 1. Lorenzo Ghiberti. Story of Jacob and Esau. Relief on the east doors of the Baptistery of St. John in Florence.

There are also the cases, when one the same forms can be interpreted at the same level by alternative ways - a kind of "visual homonymy". For example, the alternatively shifted perceptual images appear by different perceptual interpretations of one the same line configuration at the flat —as it is in cases of the "Mach figure" or "Necker cube" (see Figures $2 \& 3$ ). 


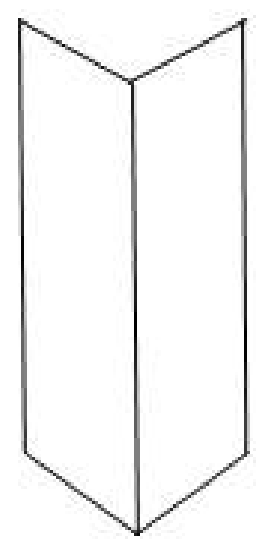

Figure 2. "Mach figure".

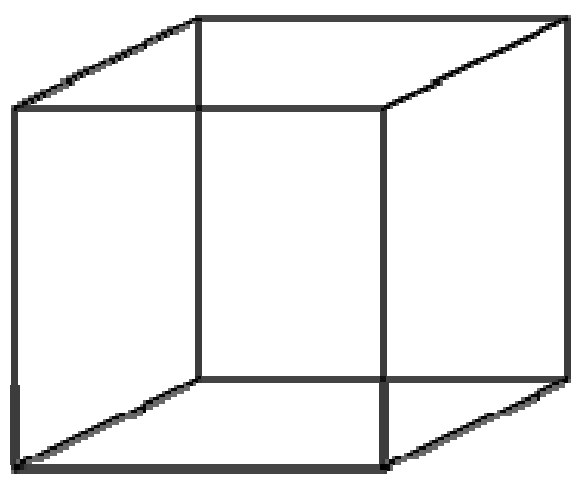

Figure 3. "Necker cube".

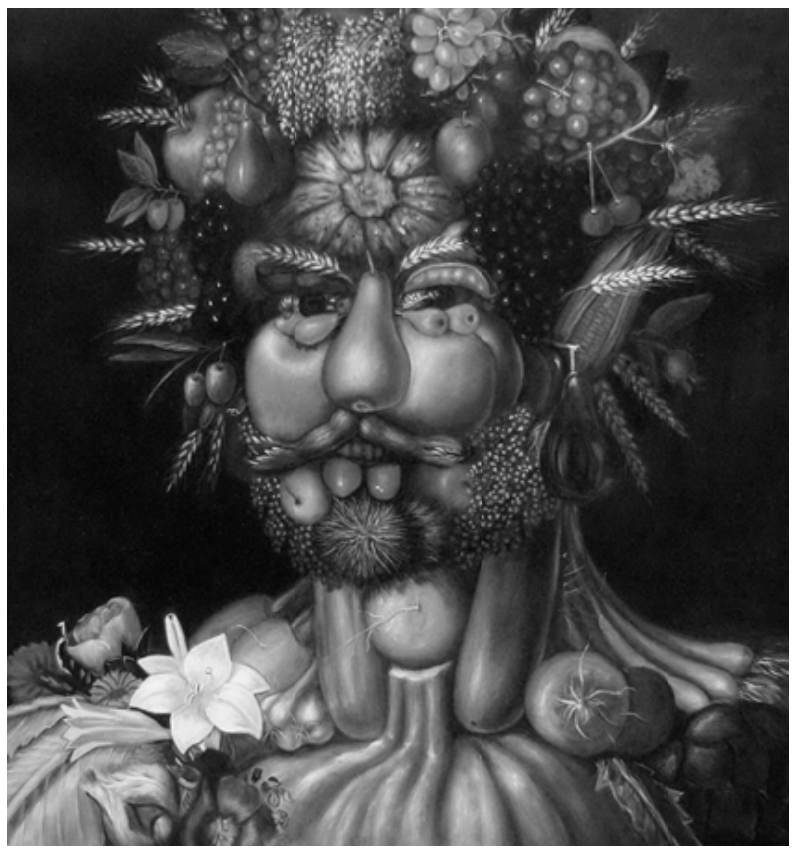

Figure 4. Giuseppe Arcimboldo. Emperor Rudolf II as Vertumnus. Oil painting on wood. 
A united participation of alternated interpretations remains also possible due to their joint in the time. Such case takes a place by an alternative recognition of depicted objects based on shifted perception of a depicting surface in dual pictures - as, for example, the allegorical paintings created by Giuseppe Arcimboldo, where the same depicted forms can be interpreted alternatively as parts of a face or as fruits as well. Painted by him, portrait of Rudolf II gives possibility even for triple alternative recognition - as a "bouquet" of diverse fruits, as an allegorical head of the Roman God of the seasons Vertumnus, and as more one head, in which the face of Rudolf II, Hole Roman Emperor, also is recognized (see Figure 4).

\section{Connections Between the Levels of Comprehension and Cognitive Codes}

Each comprehension of some new data is their including in the models of the world that were already formed by the subject at a suitable level. The information received from outside is connected at this level of comprehension with the proto-information that the subject already has-whether as results of previous experience or as some inherent structures (Tchertov, 2017; cp. concepts of Vorinformation in: Meyer-Eppler, 1959 and meta-informationin: Shreider, 1974). The proto-information contains various codes and each of them is a system of norms regulating selection, formation, interpretation, and using of received or sent data ( $\mathrm{cp}$. the extended term language, by Ch. Morris-as an inter-subject system of sign means with definite syntactic, semantic and pragmatic rules-in: Morris, 1971). If a connection between levels of comprehension occurs on a regular basis, there are norms of selecting data on a lower level and of their interpreting by the schemes of higher level, and one can talk about a code regulating this connection. The elements and structures of the lower level of comprehension are functioning in this case as components of the expression plane of such a code, whereas those of a higher level-as components of its content plane.

Particularly, schemes using for interpretation of visual sensations as of indexes of some three-dimensional forms, called by Arnheim (1974) "visual concepts", can be considered as the means of a perceptual code. This is an indexical code of natural origin, developed in the childhood along with the experience of movements in the object-spatial environment, although the diversity in this experience also depends on cultural differences (Cole \& Scribner, 1974).

Another indexical code of recognition (cp. Eco, 1976) depends more on culture-so far as the forms visible on the perceptual level are connected with generalized schemes at the level of representations. A human, who recognizes the objects as "tomato", "rose", or "lamp" using such schemes, takes thereby units from a special "visual dictionary", which regulates the connections of different perceived forms with their functional meanings (cf. Glezer, 1966). This visual "dictionary" is comparable to a verbal dictionary and has the same cultural origin, since all the units of its content plan (recognition schemes) and some units of the expression plan (reproducible forms of artifacts) are formed in culture and assimilated by individuals. In the literature, the term "meaning" is often applied to the units of such sets of schemes (see in particular: Bruner, 1973; Naisser, 1976).

Of course, the verbal languages themselves have a cultural origin, even though they are called "natural" languages. With their help, the categorization of recognizable forms is made at the conceptual level, on which these forms are included in logical classes of different degree of generality.

\section{Communicative Codes of Lover Cognitive Levels}

The mentioned codes are involved in the cognitive processes of direct comprehension of sensible objects, and not always are related to the representation of anything else in acts of inter-subject communication. Every 
such representation presumes along with direct perception, recognition, and logical categorization of a sensually given objects also their indirect, shifted comprehension.

Such comprehension is formed, particularly, by words of verbal languages that evoke ideas of both ideographic and pictographic levels. They can significate only abstract concepts like pure conventional ideograms of mathematical calculations or they can express and evoke some imaginary pictures at the "pictographic" level of imagination-like words of a literature piece. In the same time, sensations or perceptions cannot be such "psychological address" of content plane in the texts built by the words; only elements of their expression plane can be felt and perceived.

However, semiotics, which includes in its subject the codes of signal-indexical type, is able to detect those communicative means, whose content belongs to lower cognitive levels. As shown above, such semiotics with help of some psychological concepts, can describe also the means interpreted at perceptual and even sensorial levels. Such means essential differ from the verbal or similar means of object's representation and communication between subjects.

In particular, the means of depictions on the plane can represent and communicate some perceptual images from a subject to another one. Independent on an interpretation of such depictions on higher cognitive levels, their indirect, shifted comprehension supposes at least creation in the mind of a viewer of a perceptual image of represented objects - a perception of something that is absent at the depicting surface or behind it, although maybe it was available for perception of the message sender. As the means of "recording" of a probable perceptual image of an author of picture and of evoking a similar perceptual image by a receiver of the pictorial message, they are above called perceptograms. In this sense, pictograms can be understood as compact perceptograms, as long as they cause not only an abstract idea of the represented object, but also at least a reduced perception of it. However, the pictogram can be so close to a purely conventional sign that a direct link is established between the scheme of recognizing its outlines and the scheme of recognizing the object designated by it. In a perceptogram, such a connection is always mediated by the perception of an object that is yet to be recognized and categorized. The perceptogram also allows a clear perception of something or someone unrecognized, say, in portraits of an "unknown".

As it was said, an impression of similarity between the depicted and the depicting is created largely due to the similar indexes of spatial positions of depicted objects to each other and to the viewer. In order for the viewer to see "behind" of a depicting space (flat and closed) a depicted space (volumetric and open), it is necessary not to repeat this space (that would require of a three-dimensional layout), but to place on the plane its indexes, which would be sufficient for constructing of the desired perceptual image. Perspective shortenings, as well as intersections, shielding, differences in illuminated and darkened areas, contrasts and nuances of various kinds, serve to create a perceptual image of any objects. Their totality in pictures forms a complex of index means, a specific indexical text, in which their joint and concerted functions - their juxtapositions and oppositions-are essential.

The norms of formation and interpretation of such visual-spatial texts can be considered as a special type of perceptographic codes that have as their basis a natural mechanism of perception, from which some of its elements and their relationships are selected in culture. Depending on the methods of vision and creating of images accepted in the culture, one or the other code of this type is formed. All these codes belong to semiotic systems of signal-indexical type differed from the conventional sign systems, although they can regulate the processes of semiosis at more low levels. For all their dependence on culture, these systems are not entirely 
arbitrary, since they develop the various possibilities that the natural perceptual code provides them. The norms of perceptographic code can include such classic visual means as the rules of linear or aerial perspective, the organization of light and shade as indexes of spatial forms, etc. Renaissance or Baroque artists, Impressionists or Cubists, emphasizing different image possibilities and tuned to different forms of vision, thereby in fact develop different versions of the perceptographic code (See more detailed on this code: Tchertov, 2005).

Going even further, one can also find indices that cause quasi-sensory images—-shifted sensations formed by direct impact on the other senses. So, visual sensations acquire also thermal characteristics, for example, a red spot is received also as "warm", blue - as "cold", etc. Similarly, sounds can be interpreted as "high" or "low", "light" or "gloomy", etc. These are examples of synesthesia, when the sensations of some modalities cause sensations, or more precisely - quasi-sensory images — of other modalities. The connections between those and others are regulated by synesthetic codes, which are also signal-indexical semiotic systems. These codes contain the units, which can be called "sensorgrams" and be related to the same series as the ideograms, pictograms, or perceptograms.

\section{Psycho-semiotic Distinctions of Codes}

It can be seen that semiotic systems differ in "mental addresses", on which their norms specify the formation of expression and content plans. On the one hand, an expression plan of different semiotic systems can be constructed at various mental levels, and on the other hand, the levels of interpreting and constructing a "psychic substance of content" (in Loui Hjelmslev terms) can be distinguished as well. The comprehension of semiotic means at different mental levels in plane of content in cases of ideography, pictography, perceptography, or sensorography is combined differently with the psychic levels on which the plan of expression of the corresponding codes is constructed: sensorial, perceptual, or apperceptual. This gives grounds to distinguish between psycho-semiotic types of codes taking into account the special combinations of the psychic levels, on which their plans of expression and of content are formed.

In particular, the sensory-perceptual type (SP-type) will include those codes, whose expression plan is formed at the sensory level, and the content plan is formed at the level of perception. This takes place in the cases of natural perceptual code and culturally generated perceptographic codes that were considered above. Elements and structures in plane of expression in visual codes of this type are indexes of three-dimensional forms in space. These indexes are derived from the relationships between visually detected at the sensory level color spots of different configurations and intensities, and their interpretation on the perceptual level supposes of forming images of real or depicted objects located at different distances from the observer.

These perceptual images, in its turn, become the "psychic substance of expression" for recognition codes, which systematically correlate them with mental schemes of natural objects or artifacts included in human activity by certain ways. Such generalized schemes elaborated in the culture and assimilated by individuals belong to content plane of these codes and develop already on the apperceptual level of psyche. Therefore, the recognition codes linking these two levels will belong to the perceptually-apperceptual type (the PA-type).

If the recognition schemes themselves are interpreted at the level of concepts, they form a plan of expression in codes of an even higher apperceptual-conceptual type (the AC-type), which establishes systematic relationships between these schemes and their conceptual meanings.

Along with the "vertical" ways of connections between the comprehension levels as "platforms", where planes of expression and contents develop, the "horizontal" ties between elements of both these planes in frame 
of one the same level are also possible. Particularly, the recognizable signs can express not only logical concepts, but also infralogical representations, realizing the connections of the apperceptual-apperceptual type (the AA-type). Such case is, for example, as part of a complex code systems, when a recognized heraldic emblem is connected with a scheme of recognition of another object (lion, eagle, etc.) that, in its turn, is a symbol of a dynasty or of a state. Similarly, diverse synesthetic codes that connect the sensations of different modalities can be attributed to the sensory-quasi-sensorial type of codes (the SQS-type).

Each of these psycho-semiotic types includes codes that regulate both direct and shifted comprehension. For example, the interpretation of colors caught in the visual field and their outlines using the code of a sensory-perceptual type can be either direct or shifted. At the direct interpretation, particularly, the placed on a surface color spots of different shapes and sizes are perceived and recognized. At the displaced interpretation of the same lines and colors, they stimulate formation of perceptual image of some other objects in the space "behind" the picture plane that also are recognized not as paint stains. Perceptual and perceptographic codes used by these interpretations differ in that the former mediates the direct perception of the visible and is limited by the cognitive processes in the psyche of individuals, whereas the latter is developed in culture as the selected by it means of shifted perception oriented toward communication of subjects.

In cases, where shifted interpretation occurs at the level of recognition (mock-up, sculpture, and other three-dimensional images), it is attracted a scheme of an object not given to either direct or shifted perception. The choice of the necessary recognition scheme depends on the introduction of a sufficient set of perceived features as indexes of the object being represented. For example, the interpretation of masks, of layouts, of actor as another persona, etc., supposes an actualization of another recognition scheme than the scheme of directly perceived object. The index code, by which such shifted recognition is produced, as well as the code of direct recognition, will refer to the perceptually-apperceptual (PA) type.

Unlike cases of shifted perception and of shifted recognition, where semiosis and mimesis can be combined, the shifted understanding by the means of pure conventional signs is based on the relation of signification, for which mimesis is not required. For the understanding of these signs, it is no longer sufficient only to grasp them in sensations and in perceptions, but their direct recognition as units of some sign system is also necessary. This means that schemes of sign recognition should be actualized at the apperceptual level in any case and that therefore all the sign systems remain within the apperceptual-apperceptual (AA) or apperceptual-conceptual (AC) types.

The codes of diverse psycho-semiotic types differ not only by the "psychical substance" of expression and contents planes, but also by their structural organization. The codes of recognition (the PA-type) can have as their essential part a semblance of a dictionary (for example, the mentioned above "dictionary" of visual schemes used for recognition of perceived objects). Unlike them, the perceptual and perceptographic codes of SP-type have, instead of such sets of ready units, the rules of formation of perceptual images with help of indexes pointing to diverse relations between their parts. In this connection, it seems a fruitful to use differentiation of codes belonging to "lexical" and to "grammatical" types, suggested still by Ferdinand de Saussure (1977, II. 6.3).

\section{Combinations of Diverse Codes}

The more complex cases also are possible, when the shifting in the process of comprehension occurs together at different cognitive levels, and codes of various types participate in it. So, for example, unlike 
ideography, whose signs are completely arbitrary, the pictography uses simplified drawings to express more or less abstract meanings. Their shifted perception presupposes the construction of a perceptual image, which immediately correlates with the recognition scheme. In the same time, each pictogram can be considered as a recognized sign with a certain meaning and also as a picture reduced to some minimal features that give a possibility for a shifted perception of a recognizable object, because these features are enough for an actualization of its scheme saved in the memory. This scheme of recognition of a depicted object is the first meaning of the pictogram as a reproducible sign - unlike of perceptogram that always has in its content plane more or less developed perceptual image of depicted object.

However, this first meaning of the pictogram is only a condition for a manifestation of its second and main meaning that is a result of interpreting the depicted object. For example, the pictogram $\odot$ represents firstly a smiled face, because it contains enough features for actualization of a suitable scheme of recognition and due to it - for creation of a reduced perceptual image of a smiling face. With that, the same sign has the secondary meaning of salutation and some other conditional meanings. These secondary meanings can be saved, even if this pictogram will be changed by the more conventional sign :), albeit it preserves still some similarity with a smiled face. The shifted recognition of the pictogram here is supplemented with a new shifting, in which the recognized schema of the object is correlated with a new idea. In a similar way, an interpretation of heraldic emblems depicting of eagle, of lion, etc., has an actualization of their scheme of recognition as a first shifting and a nomination with these schemes of some states or towns as the second shifting already at the level of verbalized concepts. By that, the subjects denoted by one the same symbol (say, lion) are differed by ways of its depiction that are not only perceived, but also recognized differently —as, for example, lion at the emblems of cityes Lion and Dresden (see figure 5).
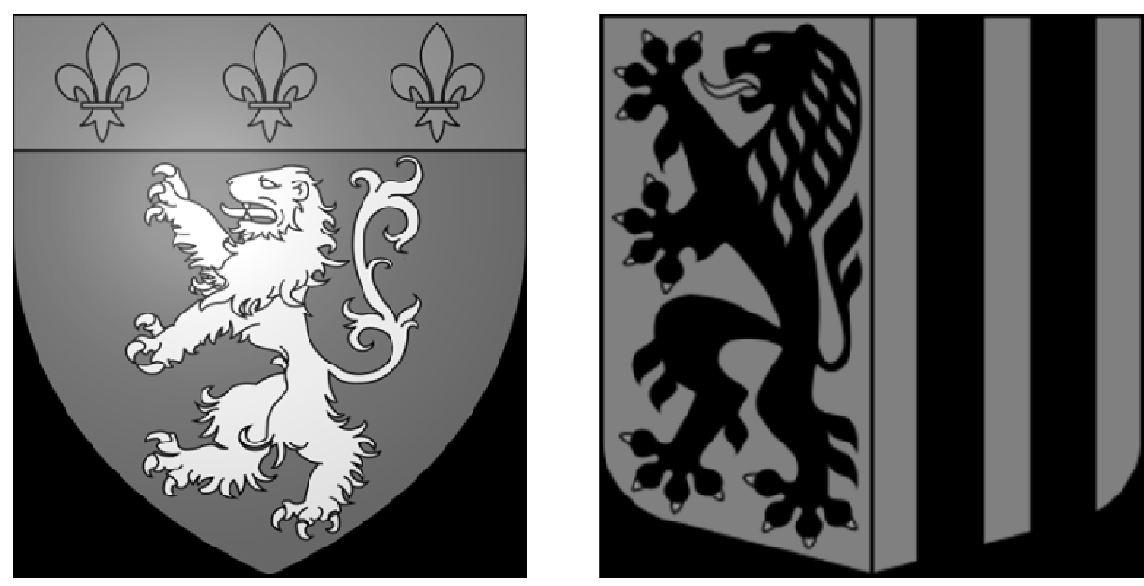

Figure 5. The pictograms of lion at emblems of cityes Lyon, France (left) and Dresden, Germany (right).

Both these examples are related to case $\mathrm{D}$ at the Scheme 1 below that helps to distinct several diverse types of shifted comprehension and codes that regulate connections between their levels.

If the scheme of recognition of a drawing shape and the scheme of recognition of a depicted object do not have such stable connection, and the pictogram is not accepted as a reproducible and recognizable sign with a constant meaning, the E-case takes a place. In this case, for example, an allegorical drawing is functioning as a more or less detailed perceptogram supposing as a supplement the shifted understanding. There are gradations between the $\mathrm{D}$ and $\mathrm{E}$ cases depending on importance of diverse comprehension levels in their mutual relations. 
Another, F-case takes a place at the comprehension of allegorical sculpture, when the direct perception of the voluminous form, for example, of a marble block, serves as the basis for the shifted recognition in it of, say, a woman with weights and a blindfold, and then for a shifted understanding of her as an allegory of Justice.

In the case of diagram, the perceived flat or three-dimensional figure should be recognized as a special form of designation, in order to be understood at the conceptual level as a visual-spatial model of the relationship between some abstract parameters (the C-case, the same as for a fully conventional sign).

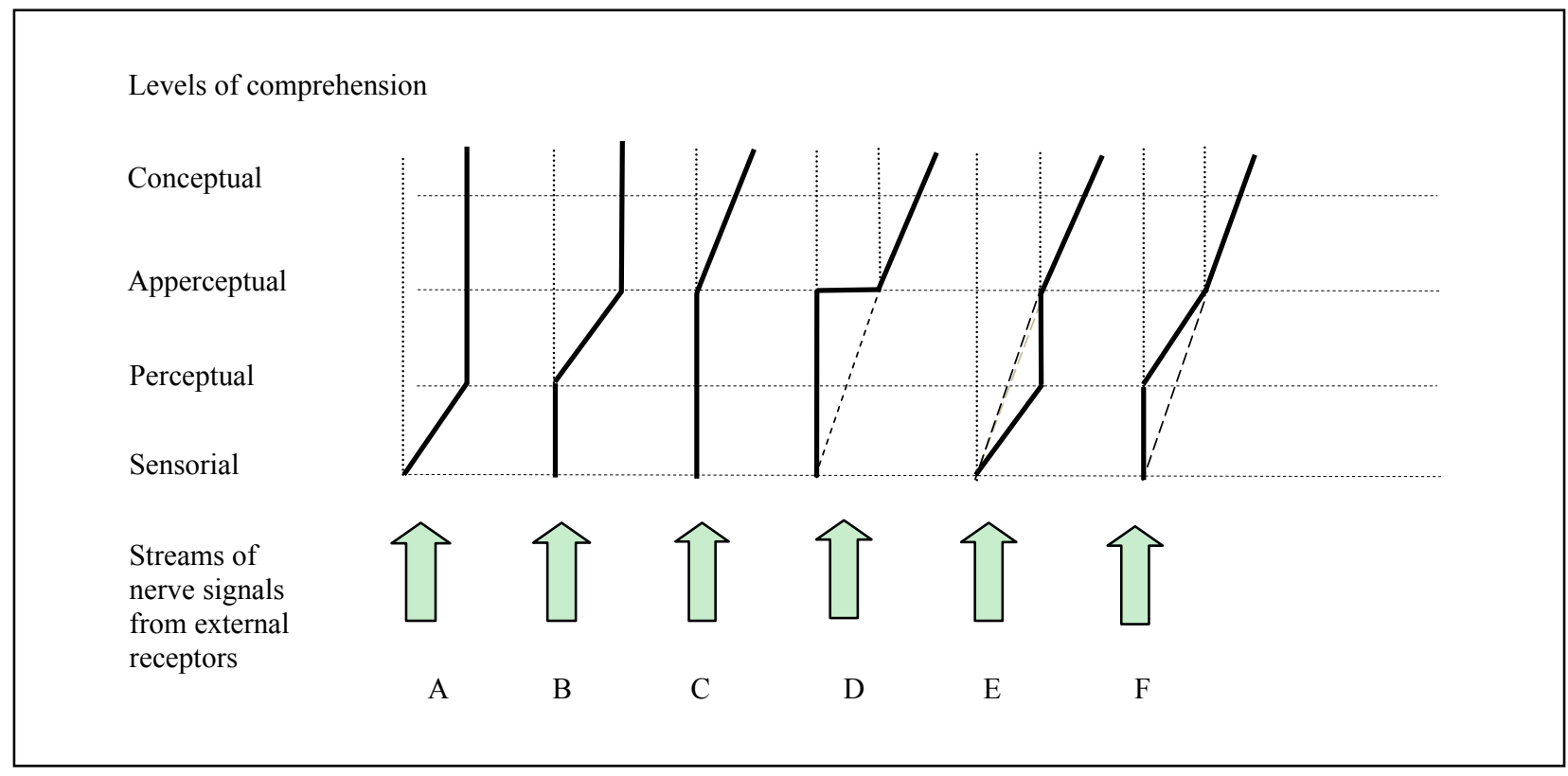

Scheme 1. Shifted comprehension at different levels with the participation of codes of various types.

A-Shifted perception of depicted objects with perceptografic codes (the SP type);

B-Shifted recognition of real objects (sculptures, dummies, mock-ups, etc.) using recognition codes (the PA type);

C-Shifted understanding of conventional signs with the help of sign systems (the AC type);

D-Shifted recognition and understanding of pictograms as known signs (the combination of the AA and AC types);

E-Shifted perception and understanding of allegorical drawings and pictograms as perceptograms (the SP and AC types);

F-Shifted recognition and understanding of allegorical sculptures (the combination of the PA and AC types).

In Scheme 1, the diagonals denote a shifting at different levels; solid lines represent "pathways" by which perception and further interpretation of sensually given objects pass in different cases of shifted comprehension; the dotted verticals indicate those levels of direct comprehension that remain the "background" for each of the cases under consideration. The dotted diagonals show a reduction in the intermediate level.

Thus, the perceptogram, pictogram, diagram, allegorical drawing, or sculpture, remaining in the class of iconic signs, according to the classification of Ch. Pierce, can be analyzed in more details by psycho-semiotic types of interpretation. The pictograms recognized as the reproducible signs should be referred for their "mental substratum" to the combinations of the AA and AC types. The more detailed and less recognizable pictograms as well as the allegorical drawings are the combinations of semiotic means of the sensory-perceptual (SP) and apperceptual-conceptual (AC) types. All three-dimensional forms intended to shifted recognition belong to the perceptually-apperceptual (PA) type. The diagrams will fall into the next apperceptual-conceptual (AC) type. Interpretation of the allegorical sculptures is the combination of the perceptual-apperceptual (PA) and apperceptual-conceptual (AC) types. 


\section{Conclusion}

Thus, the joint application of semiotic and psychologic concepts give a possibility to distinguish the psycho-semiotic types of codes that regulate the ways of shifted comprehension and communication of its results at diverse levels of psyche. On the one hand, semiotics provides the general concepts for description of communicative processes between subjects and ways of representation for them of objects. On the other hand, psychological concepts help to concretize the general semiotic schemes and to adapt them to peculiarities of non-verbal and "infralogical" means of communication.

Although the shifted comprehension is considered in the paper mainly in connection with the visual-spatial means of communication, there is no reason to believe that it is limited by them. The similar phenomena can be found in the non-verbal means of other modalities - as, for example, in music, in dance, in pantomime, etc. Taking into account that the comprehension includes, together with pure cognition, valuation and creation of planes, one can also find diverse types of shifted comprehension and corresponding codes in these spheres as well (see particularly, Tchertov, 1997).

\section{References}

Ananiev, B. G. (1977). Sensory-perceptual organization of a person. In B. G. Ananiev, On the problems of contemporary human knowledge. Moscow: Nauka [in Russian].

Arnheim, R. (1974). Art and visual perception: A psychology of the creative eye (The new version, Berkeley, Los Angeles). London: University of California Press.

Augustine, A. (1995). De doctrina Christiana (On the Teachings of Christianity). Oxford early Christian texts. R. P. H. Green (Ed. and Trans.). Oxford: Clarendon Press.

Bruner, J. (1973). Beyond the information given. Studies in psychologies of knowing. USA: W. W. Norton \& Company.

Cole, M., \& Scribner, S. (1974). Culture and thought: A psychological introduction. New York: Wiley.

De Saussure, F. (1977; 1916). Course in general linguistics. (W. Baskin, Trans.). Glasgow: Fontana/Collins.

Eco, U. (1976). A theory of semiotics. Bloomington; London: Indiana University Press.

Faworski, V. A. (1988). Lecture on drawing. In Literary-theoretical heritage (pp. 297-309). Moskow [in Russian].

Gibson, J. J. (1950). The perception of the visual world. Boston: Houghton Mifflin.

Glezer, V. D. (1966). The mechanisms of recognition of visual images. Moscow, Leningrad: Nauka.

Hjelmslev, L. (1953; 1943). Prolegomena to a theory of language (Indiana University Publications in Anthropology and Linguistics, Memoir 7). Baltimore: Waverly Press.

Meyer-Eppler, W. (1959). Grundlagen und Anwendungen der Informationstheorie (Foundations and applications of information theory). Berlin, Heidelberg: Springer Verlag.

Morris, Ch. (1971; 1938). Foundations of the theory of signs. In Ch. Morris, Writings on the general theory of signs (pp. 7-71). The Hague, Paris: Mouton.

Naisser, U. (1976). Cognition and reality: Principles and implications of cognitive psychology. San Francisco: Freeman and Compamy.

Peirce, Ch. S. (1932). Collected papers of Charles Sanders Peirce (Vol. 2): Elements of logic. Cambridge, MA: Harvard University Press.

Piaget, J., \& Inhelder, B. (1959). La genèse des structures logiques élémentaires. Classifications et sériations (The genesis of elementary logical structures. Classifications and seriations). Paris, Neuchâtel: Delachaux et Niestle.

Shreider, Y. A. (1974). Logics of sign systems. Moscow [in Russian].

Tchertov, L. (1997). The semiotization of space and dynamic codes. Semiotica, 114(3/4), 287-293. Berlin, New York: Mouton de Gruyter.

Tchertov, L. (2005). Perceptographic code in visual culture. Sign Systems Studies, 33(1), 137-158. Tartu: Tartu University Press.

Tchertov, L. (2017). Semiotics: Outlines of its history and theory. Moscow: URSS [in Russian]. 